\title{
The Value of International Collaboration in Pancreatic Cancer Research: EURECCA
}

\author{
Marc Besselink, MD, MSc, PhD (1) \\ Pancreatic and Hepatobiliary Surgery, Department of Surgery, Cancer Center Amsterdam, University of Amsterdam, \\ Amsterdam, The Netherlands
}

Pancreatic cancer is one of the worst types of cancer with a 5-year survival of $5-7 \% .^{1,2}$ Surgical resection combined with adjuvant chemotherapy is the optimal treatment option with 5-year survival rates around 20\%. In recent years, interest has grown in newer treatment strategies, such as neoadjuvant chemoradiotherapy for (borderline) resectable pancreatic cancer. Recently, the first two multicenter, randomized trials from the Republic of Korea and the Netherlands suggested a doubled R0 resection rate and benefit in overall survival benefit with this approach. ${ }^{3,4}$ It is known that fairly large variations may exist between centers, regions, and countries in treatment and outcome of patient cancer. Typically, however, granularity is lacking to explore adequately the nature of these differences.

To provide better insight into this matter, the EURECCA group, represented by Groen and coworkers from eight European countries compared baseline characteristics, and surgical and oncological treatment and outcomes, in patients with stage I and II pancreatic cancer. ${ }^{5}$ The authors obtained data from 2052 patients from national, regional, and single-center databases and audits. They observed some interesting differences. Albeit small, most baseline characteristics (e.g., age, stage, location, type of surgery) differed significantly between the various countries and centers. Even more importantly, the use of adjuvant chemotherapy, the 90-day mortality rate, and overall survival also differed significantly.

\footnotetext{
(C) Society of Surgical Oncology 2019
}

First Received: 29 November 2018;

Published Online: 4 January 2019

M. Besselink, MD, MSc, PhD

e-mail: m.g.besselink@amc.uva.nl
The results of this study should be interpreted in light of the relatively small patient numbers, ranging from 73 to 645 patients per database and varying rates of missing data between databases.

Apart from the exact findings of this work, what are the implications from this study? In which way may these findings influence our daily work? First, we should realize that differences in patient characteristics, treatment, and survival of pancreatic cancer, as in other cancers, differ much more between countries, regions, and centers than most of us would have presumed. Second, although this will not immediately influence how we treat our patients, it should influence the way that we interpret the result of clinical studies. Especially, the external validity monocenter, randomized trials in pancreatic cancer may seem less reliable. Readers should probably study the baseline characteristics table of (mono-)center studies even careful as the outcomes table and ask themselves the question: are these patients comparable to my patients? If these characteristics differ too much, the outcome of the trial may be less relevant. Third, we should attempt to design multicenter, randomized studies with participation of a large variety of centers, with varying surgical volume, from different regions, countries, and even continents. Although such multicenter, randomized trials are relative rare in pancreatic cancer, some have been performed. The ESPAC multicenter trials in Europe are a good example. These studies have provided important results on, for instance, adjuvant treatment in pancreatic cancer. ${ }^{6}$ Another, ongoing example is the DIPLOMA trial, focusing on minimally invasive distal pancreatectomy for pancreatic cancer. This study includes a large number of centers from around Europe, including Russia and the United States. ${ }^{7}$

In conclusion, much work has to be done in the field of pancreatic cancer. Patients, treatments, and outcomes of pancreatic cancer differ between centers, regions, and 
countries. This has implication for both the interpretation of (monocenter) clinical studies and the design of new randomized trials. We should be grateful to the initiative of the EURECCA for providing these insights.

\section{REFERENCES}

1. Netherlands Cancer Registry (NCR). Dutch cancer figures. http:// www.cijfersoverkanker.nl/. Accessed 1 March 2016.

2. Lepage C, Capocaccia R, Hackl M, Lemmens V, Molina E, Pierannunzio D, et al. EUROCARE-5 Working Group. Survival in patients with primary liver cancer, gallbladder and extrahepatic biliary tract cancer and pancreatic cancer in Europe 1999-2007: results of EUROCARE-5. Eur J Cancer. 2015;51:2169-78.

3. Jang JY, Han Y, Lee H, Kim SW, Kwon W, Lee KH et al. Oncological benefits of neoadjuvant chemoradiation with gemcitabine versus upfront surgery in patients with borderline resectable pancreatic cancer: a prospective, randomized, openlabel, multicenter phase 2/3 trial. Ann Surg. 2018;268(2):215-22.

4. Versteijne E, van Eijck CH, Punt CJ, Suker M, Zwinderman AH, Dohmen MA, et al. Preoperative radiochemotherapy versus immediate surgery for resectable and borderline resectable pancreatic cancer (PREOPANC trial): study protocol for a multicentre randomized controlled trial. vTrials. 2016;17(1):127.

5. Groen JV, Sibinga Mulder BG, van Eycken E, Valerianova Z, Borras JM, van der Geest LGM, et al. Differences in treatment and outcome of pancreatic adenocarcinoma stage I and II in the EURECCA Pancreas Consortium. Ann Surg Oncol. 2018;25(12):3492-501.

6. Neoptolemos JP, Palmer DH, Ghaneh P, Psarelli EE, Valle JW, Halloran CM. Comparison of adjuvant gemcitabine and capecitabine with gemcitabine monotherapy in patients with resected pancreatic cancer (ESPAC-4): a multicentre, open-label, randomised, phase 3 trial. Lancet. 2017;389(10073):1011-24.

7. van Hilst J, de Rooij T, Klompmaker S, Rawashdeh M, Aleotti F, Al-Sarireh B, et al. Minimally invasive versus open distal pancreatectomy for ductal adenocarcinoma (DIPLOMA): A PanEuropean Propensity Score Matched Study. Ann Surg. 2019;269(1):10-7.

Publisher's Note Springer Nature remains neutral with regard to jurisdictional claims in published maps and institutional affiliations. 